\title{
Fatores de risco para doenças não-transmissiveis em área metropolitana na região sul do Brasil. Prevalência e simultaneidade *
}

\section{Risk factors for non communicable diseases in a metropolitan area in South of Brazil. Prevalence and simultaneity}

Bruce B. Duncan**, Maria I. Schmidt**, Carisi A. Polanczyk***, Clécio S. Homrich****, Roger S. Rosa"*, Aloyzio C. Achutti"****

\begin{abstract}
DUNCAN, B. B. et al. Fatores de risco para doenças não-transmissíveis em área metropolitana na região sul do Brasil. Prevalência e simultaneidade. Rev. Saúde Pública, 27:143-8, 1993. Três quartos da mortalidade no Estado do Rio Grande do Sul (Brasil) ocorrem por doenças não-transmissíveis. Dentre elas as doenças cardiovasculares, por si só, correspondem a 35\% das causas de morte. Para avaliar a prevalência de fatores de risco para essas doenças, foi realizado inquérito domiciliar no período de 1986/87. Foram entrevistados 1.157 indivíduos entre 15-64 anos, residentes em setores censitários de 4 áreas docente-assistenciais do Município de Porto Alegre, RS. A prevalência padronizada de tabagismo foi de $40 \%$, hipertensão $14 \%$, obesidade $18 \%$, sedentarismo geral $47 \%$ e consumo excessivo de álcool, 7\%. Trinta e nove por cento da amostra acumulavam dois ou mais desses cinco fatores de risco, somente $22 \%$ de homens e $21 \%$ de mulheres não apresentaram esses fatores de risco. As elevadas frequências e concomitâncias desses fatores de risco alertam para sua importância em programas que visam a prevenção das doenças não-transmissíveis.
\end{abstract}

Descritores: Hipertensão, epidemiologia. Fumo, epidemiologia. Obesidade, epidemiologia. Exercício. Alcoolismo, epidemiologia.

\section{Introduçáo}

As principais doenças não-transmissíveis doenças cardiovasculares, doença pulmonar obstrutiva crônica, neoplasias, diabetes mellitus, cirrose e lesões por acidentes e violências - vêm ocupando um maior espaço no perfil da morbimortalidade de populações latino-americanas ${ }^{13}$.

No Brasil, o grupo constituído pelas doenças cardiovasculares, câncer e lesð̋es externas responde por 55\% dos 6́bitos, sendo responsável por metade do financiamento público hospitalar do pais ${ }^{2}$.

No Rio Grande do Sul, $75 \%$ das causas de morte são atribuídas às doenç̧as não-transmissiveis ${ }^{4,22}$. Comparando-se os coeficientes de mortalidade de

\footnotetext{
* Pesquisa financiada pela Organização Panamericana da Saúde, Conselho Nacional de Pesquisa (CNPq) e Fundação de Amparo à Pesquisa do Estado do Rio Grande do Sul (FAPERGS).

* Departamento de Medicina Social da Faculdade de Medicina da Universidade Federal do Rio Grande do Sul (UFRGS) - Porto Alegre, RS - Brasil.

*** Bolsista do CNPq.

**** Faculdade de Medicina da UFRGS (doutorando).

***** Departamento de Medicina Intema da UFRGS.
}

Separatas/Reprints: B. B. Duncan - Rua Ramiro Barcelos, 2600/414 - 90035-003 - Porto Alegre, RS Brasil. adultos na faixa etária 30-69 anos com os de 33 países, principalmente europeus ${ }^{5}, 27$, os do Rio Grande do Sul encontram-se entre os mais altos da série.

Apesar de haver muito ainda por ser esclarecido sobre os determinantes das doenças nãotransmissíveis ${ }^{17}$, vários fatores de risco susceptíveis a ações preventivas já estão bem definidos - entre eles fumo, hipertensão, obesidade, sedentarismo e ingestão de álcool ${ }^{9,11,23}$. Dada a alta fração etiológica atribuível a esses fatores em vários países, estão em andamento ou em fase de montagem programas nacionais de prevenção, visando à redução de suas prevalências e conseqüentemente, do risco de doenças não transmissíveis $1,3,6,12,19,20,21,24,26$.

Frente a esse cenário e ao número reduzido de dados epidemiológicos sobre as doenças nãotransmissiveis na América Latina, a Organização Panamericana da Saúde promoveu o desenvolvimento de um estudo multicêntrico em cinco países latino-americanos - Brasil, Chile, Cuba, México e Venezuela. No Brasil foram incluídos dois centros urbanos, Porto Alegre e São Paulo.

O objetivo do presente trabalho é descrever a prevalência e a simultaneidade desses fatores de risco na população adulta de Porto Alegre, de acordo com categorias de idade e sexo. 


\section{Materlal e Método}

Foi realizado inquérito domiciliar em quatro áreas docente-assistenciais de Porto Alegre, semelhantes a área urbana desta em relação à idade e sexo ${ }^{4}$. Para que a amostra destas quatro áreas melhor representasse Porto Alegre, em termos de renda domiciliar, foram escolhidos setores censitários da Fundação Instituto Brasileiro de Geografia e Estatistica (IBGE), de acordo com quartis da distribuição de renda domiciliar média de Porto Alegre, em 1980 (pontos de corte 4,8; 7,6; 11,1 salários mínimos por domicílio). Nos setores censitários escolhidos, um número determinado de domicílios foi sorteado de modo a permitir que 1/4 deles estivesse dentro dos setores censitários de cada um dos quartis da renda. Em cada domicílio, somente um morador entre 15-64 anos foi escolhido, aleatoriamente, para evitar problemas de interdependência de informação entre entrevistados.

Da amostra selecionada de 1.240 indivíduos, foram entrevistados e incluídos nas análises 1.157 (93\%). Dos 83 restantes, 33 não foram localizados após inúmeras tentativas ( 6 ou mais) em horários variados, 20 se recusaram a responder, 6 apresentavam doença mental que impedia a entrevista, 12 tiveram seus questionários extraviados e 12 foram excluídos em razão de os questionários terem sido aplicados na casa ou indivíduo errado.

Uma entrevista domiciliar foi aplicada por assistentes treinados, utilizando questionário avaliado previamente em estudo-piloto, contendo algumas questōes gerais e outras sobre os diversos fatores de risco. Durante a entrevista, era tomada a pressão arterial, o peso e a altura de cada participante.

Foi definido como fumante regular aquele que relatava fumar mais de um cigarro diariamente e como ex-fumante aquele que fumava regularmente no passado, mas não mais no presente ${ }^{25}$.

As informaçð̃es obtidas sobre freqüência, quantia e tipo de ingesta de bebidas alcoólicas ${ }^{14}$ permitiram o cálculo de gramas de álcool ingerido por semana. A classificação de consumo excessivo era feita a partir dos seguintes pontos de corte: $>=350 \mathrm{~g} / \mathrm{sem}$, para homens e $>=210 \mathrm{~g} /$ sem, para mulheres ${ }^{28}$.

Perguntas sobre atividade física no lazer, no trabalho e no caminho para o trabalho foram traduzidas de documentos do projeto North Karelia ${ }^{15}$. Foram considerados sedentários no lazer indivíduos que não exerciam atividades classificadas nas seguintes situações: pelo menos $4 \mathrm{~h}$ por semana de atividades de pequeno esforço físico (gasto metabólito de 5 - 7,5 kcal/min; por exemplo, caminhar, andar de bicicleta); no mínimo $3 \mathrm{~h}$ por semana de atividade de esforço físico maior (gasto metabólico de 7,5 - $10 \mathrm{kcal} / \mathrm{min}$; por exemplo, corrida, ginástica, futebol); ou então, no mínimo 3 dias/semana atividades de treinamento para competição (gasto metabólico maior de $10 \mathrm{kcal} /$ min). Sedentarismo geral foi atribuído ao indivíduo que não exercia atividades no trabalho que exigissem gasto metabólico superior a $5 \mathrm{kcal} / \mathrm{min}$ (como transporte de objetos pesados, subir escadas ou longas caminhadas) em pelo menos uma hora por dia; que caminhava ou andava de bicicleta (gasto metabólico de $5,5 \mathrm{kcal} / \mathrm{min}$ ) por menos que 30 min na ida e volta do trabalho; e que também era sedentário no lazer de acordo com a classificação acima.

A pressão arterial foi determinada por esfigmomanômetro aneróide através de um protocolo préestabelecido e após treinamento e certificação dos entrevistadores. Os indivíduos ficavam sentados, sem fumar ou comer por, no minimo, 5 min antes da medida. Cada esfigmomanômetro era calibrado antes de entrar em campo e periodicamente ao longo do estudo. Hipertensão arterial foi definida por um valor médio das duas leituras maior ou igual a $160 \mathrm{mmHg}$, para a pressão sistólica, e ou maior ou igual a $95 \mathrm{mmHg}$, para a pressão diastólica; ou então pelo relato de uso de medicação antihipertensiva.

Peso e altura foram medidos através de um protocolo padronizado com treinamento e certificação prévia dos entrevistadores. A medida do peso foi introduzida após o início das atividades de campo, de modo que o peso foi medido somente em 699 indivíduos (60\% da amostra), sendo utilizado o peso referido nos demais 458 indivíduos. A comparação entre peso medido e peso referido mostrou excelente correlação $(r=97 \%)^{29}$. $O$ índice de massa corporal foi calculado dividindo-se o peso (em $\mathrm{kg}$ ) pelo quadrado da altura (em metros). Os pontos de corte para obesidade foram aqueles adotados pelo National Health and Examination Survey-II ${ }^{16}$, ou seja, de 27,3 e 27,8 para mulheres e homens, respectivamente.

As prevalências gerais foram ajustadas por idade, de maneira direta ${ }^{10}$, utilizando como padrão a população de Porto Alegre?.

Intervalos de confiança para as prevalências relatadas (indicados entre parênteses) foram calculados como: prevalência +/- 1,96 (erro padrão), onde 0 erro padrão é calculado como a raiz quadrada da expressão $\mathrm{p}(1-\mathrm{p}) / \mathrm{n}$, onde $\mathrm{p}=$ prevalência do fatơr em questão e $\mathbf{n}=$ número de indivíduos examinados ${ }^{8}$.

\section{Resultados}

Dos 1.157 indivíduos efetivamente estudados, $62 \%$ eram mulheres e $20 \%$ eram pretos ou mula- 
tos. A distribuição desses indivíduos de acordo com alcance escolar mostra que $6 \%$ deles não tinham completado a primeira série, $53 \%$ cursaram pelo menos uma das demais séries do primeiro grau, $19 \%$ do segundo grau e $22 \%$ de um curso superior. A mediana da renda per capita era de 1,9 salários mínimos, sendo que $10 \%$ ganhavam mais que 6,1 (decil superior) e $10 \%$ menos que 0,5 (decil inferior).

A Tabela 1 mostra as prevalências brutas e seus intervalos de confiança de $95 \%$ dos fatores de risco estudados, separadamente para homens e mulheres. Chamam à atenção as elevadas prevalências de todos os fatores, em ambos os sexos, com exceção do consumo de álcool em mulheres, em que a prevalência foi de $3 \%$. Homens e mulheres apresentam diferenças nítidas nessas prevalências, como evidenciado pela não superposição dos intervalos de confiança, sendo a única exceção desse quadro, a prevalência de hipertensão.

Tabela 1. Prevalência dos fatores de risco para doenças crónicas em homens e mulheres. Porto Alegre, 1986-7.

\begin{tabular}{lcc}
\hline Fatores de Risco & $\begin{array}{c}\text { Homens } \\
(n=437) \\
\%(I C ~ 95 \%)\end{array}$ & $\begin{array}{c}\text { Mulheres } \\
(n=700) \\
\%(I C 95 \%)\end{array}$ \\
\hline $\begin{array}{l}\text { Hipertensão } \\
\text { Fumo }\end{array}$ & $15(12-18)$ & $15(12-18)$ \\
Consumo excessivo & $52(47-57)$ & $33(30-36)$ \\
de Álcool & $13(10-16)$ & $3(2-4)$ \\
Obesidade & $16(13-19)$ & $24(21-27)$ \\
Sedentarismo Geral & $38(33-43)$ & $58(54-62)$ \\
Sedentarismo no Lazer & $69(65-73)$ & $82(79-85)$ \\
\hline
\end{tabular}

\section{IC - Intervalo de confiança.}

As prevalências ajustadas por idade, utilizando padrões etários de Porto Alegre, não são muito diferentes das encontradas na amostra estudada: para homens, a prevalência ajustada de hipertensão foi de $14 \%$, de fumo $50 \%$, de obesidade $14 \%$, de consumo excessivo de álcool $12 \%$ e de sedentarismo geral $36 \%$, sendo a de sedentarismo no lazer 67\%; para mulheres, a prevalência ajustada de hipertensão foi de $14 \%$, de fumo $32 \%$, de obesidade $22 \%$, de consumo excessivo de álcool $3 \%$ e de sedentarismo geral $57 \%$, sendo a de sedentarismo no lazer $80 \%$.

As prevalências dos vários fatores de risco, em homens e mulheres, de acordo com grupos etários são apresentadas nas Figuras 1 e 2 . Observa-se uma tendência para aumento nas prevalências dos fatores de risco das faixas etárias mais jovens para as faixas de meia idade, com tendência à queda nas idades mais avançadas. $\mathrm{O}$ aumento com a idade é particularmente evidente nas prevalências de obesidade e hipertensão entre as mulheres.
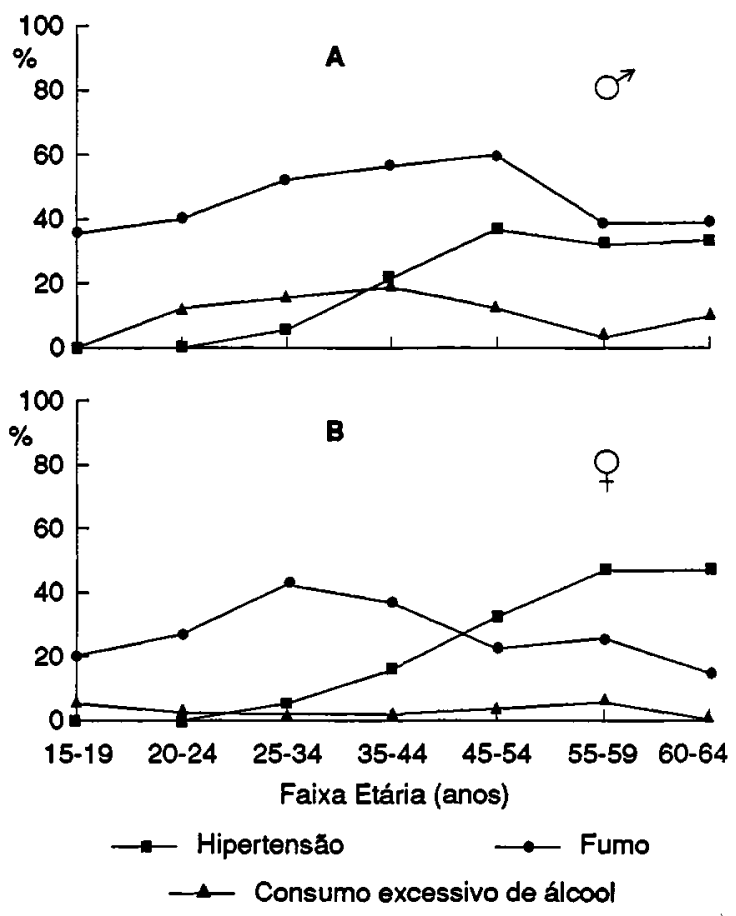

Figura 1. (A — B) Prevalência de hipertensão, fumo e consumo excessivo de álcool, de acordo com faixa etária, em homens $\theta$ mulheres, como fatores de risco para doenças crônicas. Porto Alegre, 1986-7.
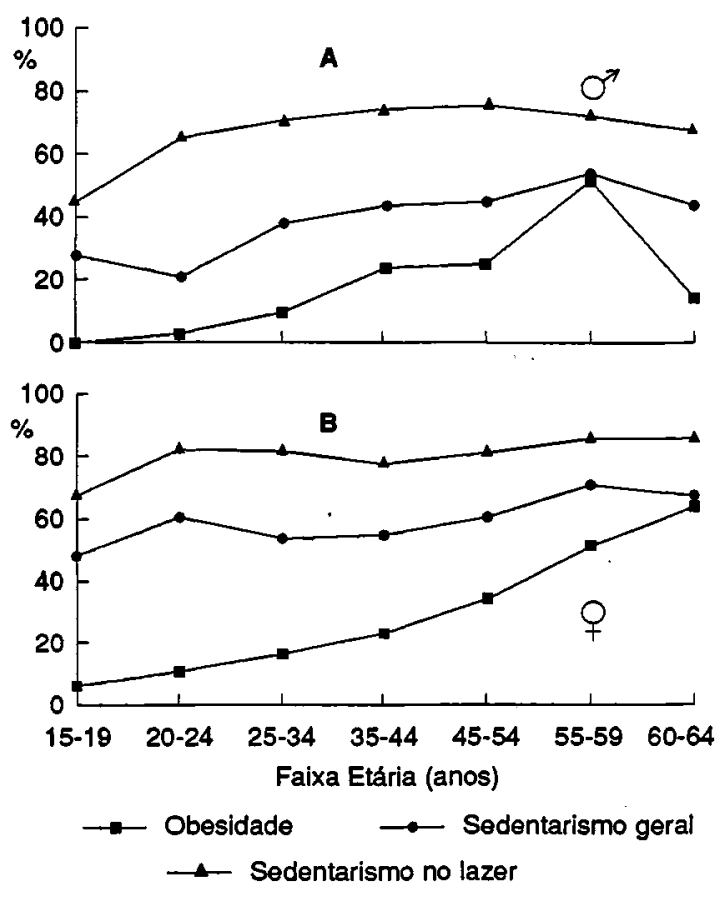

Figura 2. (A e B) Prevalências de obesidade, sedentarismo geral e sedentarismo no lazer, de acordo com faixa etária, em homens e mulheres como fatores de risco para doenças crônicas. Porto Alegre, 1986-7. 
A Tabela 2 apresenta a distribuição dos indivíduos, de acordo com o número de fatores de risco acumulados, separadamente para homens e mulheres. Observa-se grande similaridade entre estes dois grupos, sendo que $39 \%$ de ambos acumulavam dois ou mais fatores de risco. Os fatores mais freqüentemente acumulados em ambos os grupos, eram o sedentarismo e o fumo.

Tabela 2. Distribuiçăo dos individuos de acordo com o número de fatores de risco acumulados para doenças crônicas. Porto Alegre, 1986-7.

\begin{tabular}{ccc}
\hline $\begin{array}{c}\text { Números de } \\
\text { Fatores de Risco }\end{array}$ & $\begin{array}{c}\text { Homens } \\
n=426^{*} \\
\%(I C 95 \%)\end{array}$ & $\begin{array}{c}\text { Mulheres } \\
n=702^{*} \\
\%(I C 95 \%)\end{array}$ \\
\hline 0 & $22(18-26)$ & $21(18-24)$ \\
1 & $38(33-43)$ & $39(35-43)$ \\
2 & $26(22-30)$ & $28(25-31)$ \\
3 & $10(7-13)$ & $10(8-12)$ \\
4 & $3(1-5)$ & $1(0,3-1,7)$ \\
5 & $0,2(0-0,6)$ & 0 \\
\hline
\end{tabular}

- Números menores do que os totais de indivíduos estudados, por informação incompleta sobre um ou mais dos 5 latores de risco.

IC - Intervalo de confiança.

A Figura 3, para homens, e a Figura 4, para mulheres, ilustram essas distribuiçбes de acúmulo, de acordo com a faixa etária.

Entre os homens, na faixa etária 15-19 anos, $40 \%$ não apresentavam nenhum fator de risco, cerca de $51 \%$, um fator, e somente $9 \%$, dois fatores. Este quadro modifica-se dramaticamente com o avanço da idade. Da faixa 35-44 anos até a faixa 55-59 anos, mais que 50\% apresentavam dois ou mais fatores de risco. $\mathrm{Na}$ faixa $45-54$, somente $9 \%$ não apresentavam fator algum, $30 \%$ um fator, $39 \%$ dois, $14 \%$ três e $7 \%$ quatro fatores de risco.

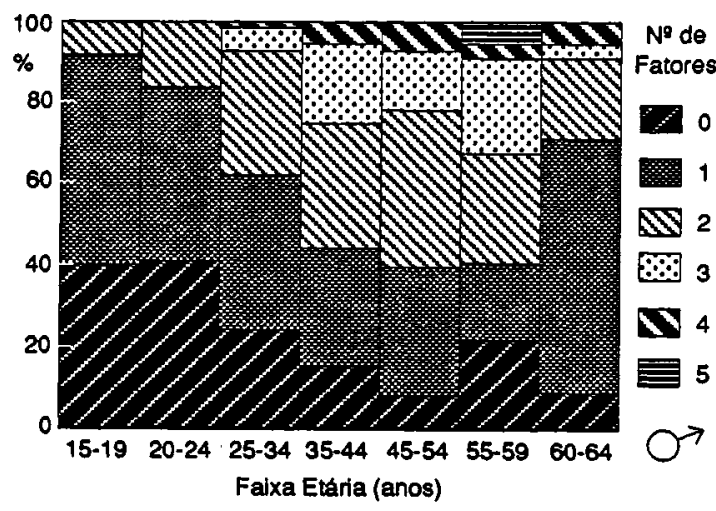

Figura 3. Percentual de acúmulo de fatores de risco para doenças nāo-transmíssiveis em homens, conforme idade. Porto Alegre, 1986-7

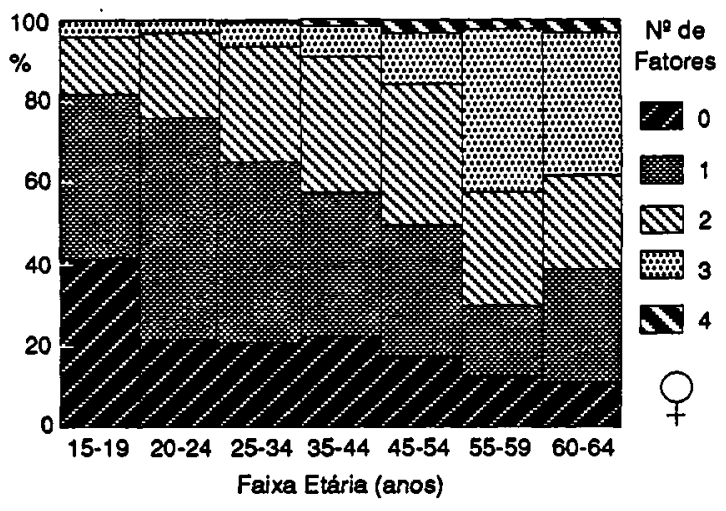

Figura 4. Percentual de acúmulo de fatores de risco para doenças nāo-transmissiveis em mulheres, conforme idade. Porto Alegre, 1986-7.

Acima de 55 anos observa-se uma diminuição da concomitância de fatores de risco.

O quadro nas mulheres é semelhante, com $81 \%$ da população de 15-19 anos apresentando zero a um fator e com um notável aumento do acúmulo de fatores com a idade. Nas mulheres, porém, o aumento com a idade é pouco menos acentuado. A redução nas prevalências nas faixas etárias mais avançadas é menos evidente nas mulheres, manifestando-se apenas na faixa etária de 60-64 anos. Assim, nas faixas mais avançadas, a concomitância é muito maior em mulheres.

\section{Discussáo}

O presente estudo foi planejado como uma linha de base para avaliação de ações comunitárias desenvolvidas a partir de quatro áreas docenteassistenciais de Porto Alegre. Assim, não foi delineado para constituir uma amostra representativa da população adulta de Porto Alegre, embora uma semelhança com esta fosse buscada. Análises comparativas mostram boa semelhança, em termos socioeconômicos, entre a amostra efetivamente estudada e a população de 15-64 anos de Porto Ale$\mathrm{gre}^{4}$. Pequenas diferenças por idade e sexo foram encontradas entre a amostra e a população de Porto Alegre. No entanto, as prevalências ajustadas com o padrão etário de Porto Alegre são muito semelhantes às da amostra. Não havendo razão específica para suspeitar que os dados deste estudo não sejam comparáveis aos da população adulta de Porto Alegre, nessa faixa etária, é possível generalizá-los para a área urbana de Porto Alegre, usando os coeficientes específicos para cada sexo.

As altas prevalências aqui encontradas são motivo de preocupação para os planejadores de saúde. Com exceção do consumo excessivo de álcool em mulheres, todos os demais fatores esti- 
veram presentes, em homens e mulheres, com freqüências superiores a $10 \%$. Alguns, como fumo em homens e sedentarismo no lazer em ambos os sexos, acometeram mais da metade da população estudada. Como 0 acúmulo de fatores cresce dramaticamente com a idade, a partir de 35 anos para homens e 45 anos para mulheres, mais da metade da população acumulava dois ou mais fatores. $O$ decréscimo do acúmulo mais acentuado para homens, visto a partir dos 60 anos, é digno de nota, embora baseado em número pequeno de observações. É possível que 'a mortalidade antes dos 60 anos seja seletiva para homens com maior acúmulo, de modo que em um estudo transversal como este, eles entram com menor prevalência.

As prevalências aqui relatadas são elevadas também quando comparadas com dados internacionais. Considerando hipertensão, fumo e obesidade, as prevalências deste estudo, com exceção da obesidade em homens, situam-se acima da mediana das prevalências relatadas pelos centros do Projeto MONICA ${ }^{15}$. Em homens, a prevalência de fumo aproxima o topo da lista de prevalências desses centros ${ }^{18}$.

A presença massiva desses fatores demanda programas de prevenção comunitários e clínicos para as doenças cardiovasculares e outras doenças não-transmissiveis - o que vem sendo adotado, de uma forma ou de outra, em vários outros países. Nesse sentido, é lastimável a dificuldade em se reconhecer a crescente importância das doenças nãotransmissíveis no Brasil, e a baixa prioridade, no setor de saúde, atribuída para os programas comunitários visando sua prevenção. Isso adquire dimensão ainda maior no contex to em que crescente proporção dos parcos recursos disponiveis para saúde é dispendida no diagnóstico e manejo dessas patologias, nos níveis secundário e terciário do setor saúde.

DUNCAN, B. B. et al. [Risk factors for non comunicable diseases in metropolitan area in South of Brazil. Prevalence and simultaneity]. Rev. Saúde Pública, 27: 143-8, 1993. Three-quarters of deaths in Rio Grande do Sul State, Brazil, are due to non-communicable diseases - cardiovascular diseases, alone, being responsible for $35 \%$ of them. To evaluate the prevalence of risk factors for these diseases, a household survey of 1,157 randomly sampled individuals between 15 and 64 years of age was undertaken in 1986 and 1987 in census tracts of 4 areas of the city of Porto Alegre. The ageand sex-adjusted prevalence of smoking was $40 \%$, hypertension $14 \%$, obesity $18 \%$, overall sedentary lifestyle $47 \%$, and excessive alcohol consumption $7 \%$. Thirty-nine percent of the sample presented two or more of these five risk factors, and only $22 \%$ of men and $21 \%$ of women had none of them. The high fre- quencies and simultaneous presence of these risk factors indicate their importance for programs aimed at the prevention of non-communicable diseases and the promotion of adult health.

Keywords: Hypertension, epidemiology. Smoking, epidemiology. Obesity, epidemiology. Alcoholism, epidemiology.

\section{Referências Bibllográficas}

1. BLACKBURN, H.; LUEPKER, R. V.; KLINE, F. G. The Minnesota Heart Health Program: a research and demonstration project in cardiovascular disease prevention. In: Matarazzo, J. D. Behavioral health: a handbook of health enhancement disease prevention. New York, John Wiley Sons, 1984. p. 1171-8.

2. BRISCOE, J. Brasil: the new challenge of adult health: $a$ World Bank Country Stucty. Washington, The World Bank, 1989.

3. CARLETON, R. A.; LASATER, T. M.; ASSAF, A. R. The Pawtucket Heart Health Program: a experiment in population-based disease prevention. Rhode. Island Med. J., 70: 533-8, 1987.

4. DUNCAN, B. B. As desigualdades sociais na distribuição de fatores de risco para doenças não-transmissíveis. Porto Alegre, 1991. [Tese de Doutorado - Universidade Federal do Rio Grande do Sul].

5. DUNCAN, B. B.; SCHMDT, M. I.; POLANCZYK, C. A.; MENGUE, S. S. Altos coeficientes de mortalidade em populações brasileiras: uma comparação intemacional. Rev. Ass. Med. Bras., 38: 138-44, 1992.

6. FARQUHAR, J. W.; FORTMANN, S. P; MACCOBY, N. The Stanford Five-City Project: design and methods. Am. J. Epidemiol., 122: 323-34, 1985.

7. FUNDAÇÃO IBGE. Pesquisa nacional por amostra de do. micllios - 1985. Riode Janeiro, 1985. v.9, t. 2, p. 274.

8. GARDNER, M. J. \& ALTMAN, D. G. Statistics with confidence: confidence intervals and statistical guidelines. London, Br.Med. J., 299: 690, 1989.

9. KAPLAN, N. M. \& STAMLER, J. Prevention of coronary heart disease pratical management of risk factors. Philadelphia, W. B. Saunders Co, 1983.

10. LAURENTI, R.; JORGE, M. H. P. M.; LEBRÃO, M. L.; GOTLIEB, S. L. D. Estatística de saude. São Paulo, EPU, 1985.

11. LEPARSKI, E. The prevention of noncommunicable disease: experiences and prospects. Copenhagen, World Health Organization, 1987. (ICP/NCD 028/6).

12. LEPARSKI, E. \& NÜSSEL, E. Protocol and guidelines for monitoring and evaluation procedures. Countrywide Integrated Noncommunicable Diseases Intervention Program (CINDI). Heidelberg, Springer-Verlag, 1987.

13. LITVAK, J.; RUIZ, L.; RESTREPO, H. E.; McALLIS. TER, A. The growing noncommunicable disease burden, a challenge for the countries of the Americas. Bull. Pan. Am. Health. Organ., 21: 156-71, 1987.

14. McALLISTER, A. Investigación de la comunidad para prevención de enfermedades no comunicables; PAHO Version. San Antonio University of Texas, 1985. [Mimiografado].

15. NATIONAL PUBLIC HEALTH INSTITUTE, HELSINKI, FINLAND. Monitoring of cardiovascular diseases and their risk factors at the community level (Finnish part of a Joint WHO Study). The Monica Project. Geneva, World Health Organization, MNC/MOO/ Appendix I, July, 1982. 
16. NIH CONSENSUS DEVELOPMENT PANEL ON THE HEALTH CONSEQUENCES OF OBESITY. Health implications of obesity, National Institutes of Health Consensus Development Conference Statement. Ann. Intern. Med. 103: 1073-7, 1985.

17. ORGANZACION PANAMERICANA DE LA SALUD. Oficina de Coordinacion de Investigaciones. $\mathrm{La}$ investigación en salud: política y prioridades de OPS. Bol. Epidemiol. Organ. Panam. Salud, 6: 1-3, 1983.

18. POLANCZYK, C. A.; DUNCAN, B. B.; SCHMIDT, M. I.; ACHUTTI, A. C.; KUMMER, S. C.; MAIA, A. L. G. Prevalência e simultaneidade de fatores de risco para doenças năo-transmissíveis em Porto Alegre. In: Congresso Brasileiro de Epidemiologia, 12. Campinas, 1990. Programa Cientifico. Campinas, 1990: resumo 98.

19. PUSKA, P. Comprehensive cardiovascular community control programmes in Europe. Copenhagen, World Health Organization, 1988. (EURO Reports and Studies $\mathrm{n}^{2} .106$ ).

20. PUSKA, P.; NISSIMEN, A.; SALONEN, J. T.; TOUMILEHTO, J. Ten years of the North Karelia Project: results with community-based prevention of coronary heart disease. Scan. J. Soc. Med., 3: 65-8, 1983.

21. PUSKA, P.; TOUMIIEHTO, J.; SALONEN, J. T. et al. Community control of cardiovascular disease: the North Karelia Project. Copenhagen, World Health Organization. Regional Office for Europe, 1981.

22. SECRETARIA DA SAUDE E DO MEIO AMBIENTERIO GRANDE DO SUL. Estatistica de saúde: mortalidade, 1989. Porto Alegre, 1990. v. 15.

23. WHO EXPERT COMMITTEE ON COMMUNITY PREVENTION AND CONTROL OF CARDIOVASCU-
LAR DISEASE. Geneva, 1984. Report. Geneva, 1986. (WHO Tech. Rep. Ser., 732).

24. WORLD HEALTH ORGANIZATION. Developing of methodology for prevention and control of cardiovascular disease in primary health care in development countries; Report of a meeting of investigators, Geneva, 1985. (CVD/85.5).

25. WORLD HEALTH ORGANIZATION. Guidelines for the conduct of tobacco smoking surveys of the general population - Report of a WHO meeting held in Helsinki, Finland 29 November-4 December 1982. Geneva, 1983. (Publication WHO/SMO/83.4).

26. WORLD HEALTH ORGANIZATION. Integrated Project for Community Health in noncommunicable diseases (Interhealth). Guidelines for a core protocol. Geneva, 1987. (WHO/NCD/87.2).

27. UEMURA, $K$. \& PISA, $Z$. Trends in cardiovascular disease mortality in industrialized countries since 1950 . World. Health Statist. Quart., 41: 155-78, 1988.

28. WALLACE, P.; CULTLER, S.; HAINES, A. Randomized controlled trial of general practitioner intervention in patients with excessive alcohol consumption. Br. Med. $J ., 297: 663-8,1988$.

29. ZIMMER, P. M.; PELLANDA, L. C.; TAVARES, M. R. G.; DUNCAN, B. B.; SCHMIDT, M. I. Validade do peso referido para uso em estudos epidemiológicos. In: Congresso Brasileiro de Diabetes, $7^{\circ}$, Guarapari, 1989. Programa Científico. p. 48.

Recebido para publicaçäo em 8.9.1992 Aprovado para publicação em 20.11.1992 